\title{
The effect of opium on severity of COVID-19 infection:An original study from Iran
}

\author{
Taghi Riahi ${ }^{1}$, Afsaneh Sadeghzadeh-Bazargan ${ }^{2} * \mathbb{1}$, Sima Shokri $^{3}$, Davoud Ahmadvand ${ }^{4}$, Babak Hassanlouei ${ }^{5}$, \\ Amir Baghestani ${ }^{6}$, Ali Khazaeian ${ }^{6}$, Forough Seifi Gharabaghloo ${ }^{6}$, Morteza Hassanzadeh ${ }^{1}$, Azadeh Goodarzi ${ }^{2} *(1)$
}

Received: 23 Oct $2020 \quad$ Published: 8 Sep 2021

\begin{abstract}
Background: The COVID-19 infection is a novel virus without any specific targeted therapies; thus, focusing on primary epidemiologic concerns, preventive strategies, risk factors, exacerbation factors, and mortality-related factors are of great importance to better control this disorder. There are some controversies about the factors associated with COVID-19 in different theories, and addiction is no exception.

Methods: We conducted a large cross-sectional study of 513 hospitalized Iranian patients with COVID-19 infection to evaluate the severity of disease courses in patients with or without history of opium addiction. We recorded these datia retrospectively after patients' discharge from the hospital. For the quantitative data, we used independent-samples $t$ and Mann-Whitney tests. The qualitative data were calculated using Fisher exact and chi-square tests in IBM SPSS Statistics Version 22. Also, $p<0.105$ was considered statistically significant.
\end{abstract}

Results: There was no significant difference regarding mean days of hospitalization in opium positive and negative groups ( $7.95 \pm 8.39$ vs $8.35 \pm 5.11$, respectively) ( $\mathrm{p}=0.771)$; however, the need for intensive care unit (ICU) admission was significantly higher in the opium positive group ( $36 \%$ vs $11 \%)(\mathrm{p}=0.005)$. The mean days of ICU stay was significantly higher in the opium positive group $(2.36 \pm 3.81$ vs $0.86 \pm 2.90)(\mathrm{p}=0.026)$. The percentage of febrile patients, anosmia/hyposmia, and dysgeusia at the initiation of hospitalization was significantly lower in the opium positive group ( $39 \%$ vs $66 \% ; 8 \%$ vs $23 \%$; $8 \%$ vs; $20 \%$, respectively) ( $\mathrm{p}=0.002$, 0.018 , and .031 , respectively). In the laboratory tests, only the white blood cell (WBC) count and the segmented cells were higher in the opium positive group ( $10.1 \pm 6.60$ vs $7.38 \pm 4.14$ and $73 \pm 20.47$ vs $56.5 \pm 32.60$, respectively) ( $p=0.018$ and .001 , respectively) and lymphocytes were lower in the opium positive $(15.60 \pm 8.25$ vs $18.70 \pm 10.12)(\mathrm{p}=0.048)$. Opium addicts had a significantly lower rate of azithromycin and lopinavir/ritonavir prescription in their initiation therapy ( $19 \%$ vs $34 \%$, and $47 \%$ vs $70 \%$, respectively) ( $p=0.038$ and 0.012 , respectively).

Conclusion: Opium addict patients with COVID infection may be more febrile and experience more disease-specific symptoms and more severe disease course. These patients may show more evidence of laboratory inflammation and probable superinfections, so may manage with more caution and somehow different therapeutic regimen.

Keywords:Corona, COVID-19, SARS-CoV-2, Opium, Substance, Addiction, Drug Abuse, Severity, Outcome

Conflicts of Interest: None declared

Funding:None

*This work has been published underCC BY-NC-SA 1.0 license.

Copyright $₫$ Iran University of Medical Sciences

Cite this article as: Riahi T, Sadeghzadeh-Bazargan A, Shokri S, Ahmadvand D, Hassanlouei B, Baghestani A, Khazaeian A, Seifi Gharabaghloo F, Hassanzadeh M, Goodarzi A. The effect of opium on severity of COVID-19 infection:An original study from Iran.Med J Islam Repub Iran. 2021(8 Sep);35:115. https://doi.org/10.47176/mjiri.35.115

Corresponding author: Dr Azadeh Goodarzi, goodarzi.a@iums.ac.ir DrAfsaneh Sadeghzadeh-Bazargan, sadeghzadeh.a@iums.ac.ir

1. Department of Internal Medicine, School of Medicine, Rasool Akram Medical Complex, Iran University of Medical Sciences, Tehran, Iran

2. Department of Dermatology, Rasool Akram Medical Complex, Iran University of Medical Sciences, Tehran, Iran

3. Department of Allergy and Clinical Immunology, Rasool Akram Medical Complex, Iran University of Medical Sciences, Tehran, Iran

4. Department of Medical Laboratory Sciences, School of Allied Medical Sciences, Iran University of Medical Sciences, Tehran, Iran

5. Department of Epidemiology, School of Public Health, Iran University of Medical Sciences, Tehran, Iran

6. Department of General Medicine, Rasool Akram Medical Complex, Iran University of Medical Sciences, Tehran, Iran $\uparrow$ What is "already known" in this topic:

The COVID-19 infection is a novel virus without any specific targeted therapies. There was more evidence against any protective role of addiction on COVID-19 infection or experiencing any better course of disorder in an addicted person, so that most studies have reported addiction as a triggering or worsening factor of COVID-19.

\section{$\rightarrow$ What this article adds:}

Opium-addicted patients with COVID infection may be more febrile and experience more disease-specific symptoms and more severe disease course. These patients may reveal more evidence of laboratory inflammation and probable superinfections. 


\section{Introduction}

The COVID-19 infection is a novel virus that mainly targets the respiratory system via specific receptors without any targeted therapies; thus, focusing on primary epidemiologic concerns, preventive strategies, risk factors, exacerbation factors, and mortality-related factors are of great importance needing to be evaluated (1). One of the hot topics with somehow controversies is the effect of smoking and addiction on the infection rate and disease consequences of COVID-19 in this pandemic area.

The negative effect of smoking on disease severity and mortality has been shown in several studies in the different viral infections, such as influenza, MERS-CoV, and recently in COVID-19 $(2,3)$. This adverse effect may be due to the impact of smoking on immune system function, more exposure to contagious infections or the direct destructive effect of smoking on lung tissue. On the other hand, this effect has been observed in patients with both current and previous history of smoking (3).

However, there are controversial findings of the effect of opium and other substances on the prevalence, severity, and mortality rate of COVID-19. In a study from Iran, Khoshab et al reported the protective effect of opium addiction against COVID-19 infection, because none of their COVID-19infectedpatients had a history of opium addiction (4).

Another study from Iran showed a higher mortality rate of COVID-19 in opium-addicted patients compared with nonaddicted population (5).

In all relevant studies, the adverse effect of smoking in final outcomes of COVID-19 is evident. Up to September 2020, when we were writing this article, there was more evidence against any protective role of addiction on COVID-19 infection or experiencing any better course of disorder in an addicted person, so that most studies have reported the addiction as a triggering or worsening factor of COVID-19 (2, 3, 5-8). However, due to some overall controversies, especially about addiction, for more exact and more complete discussion (on the effect of opium addiction in infection risk and disease-consequence), we need more well-designed controlled studies. In this large cross-sectional study from Iran, we aimed to evaluate the effects of opium addiction on the severity of incoming COVID-19 infection.

\section{Methods}

This analytical cross-sectional study was conducted on 549 Iranian patients affected by COVID-19 between March to May 2020 who were hospitalized in RasoolAkram Medical Complex affiliated to Iran University of Medical Sciences, Tehran, Iran. Their diagnoses were approved by a positive nasopharynx RT-PCR test;or in the case of negative polymerase chain reaction (PCR) test, based on very suggestive computed tomography (CT) imaging for COVID-19 scored by the CO-RADS classification system (6). Of 549 patients, 36 cases (6.5\%) had a positive history of addiction with a focus on opium addiction. This history was taken by an expert specialist who managed the patients and recorded their data. We tried to compare the severity of COVID-19 in patients with or without opium addiction history. However, we did not consider smokers in this comparison. The main outcomes of this study for comparison between the 2 groups (which were considered indirectly to be associated with the overall severity score of the disease) were the mean days of hospitalization, the necessity for intensive care unit (ICU) admission, the average days of staying in the ICU, and the need to take second-line therapeutic options. For both groups, we assessed demographic data of the disease and the patients, laboratory tests, and treatment protocols, completely. Continuous variables were presented as mean and SD, and for the quantitative data, independent samples $t$ and Mann-Whitney tests were used.The qualitative data were calculated using the Fisher exact and chi-square test in IBM SPSS Statistics Version 22; and $p<0.05$ was considered statistically significant. The ethical code of the large cohort study of RasoolAkram Medical Complex from which the data for this study were extracted was as follows: IR.IUMS.REC.1399.759.

\section{Results}

Demographic data of the study participates has is presented in Table 1.There was no significant difference between the groups regarding mean days of hospitalization; however, the need for ICU admission was significantly higher in the opium positive group (36.1\% vs $11.3 \%$ $(p=0.005)$; the mean days of ICU stay was higher in the opium positive group $(2.36 \pm 3.81$ vs $0.86 \pm 2.90)(\mathrm{p}=0.026)$. The percentage of febrile patients, anosmia/hyposmia, and dysgeusia at the initiation of hospitalization was lower in the opium positive group (39\% vs $66 \% ; 8 \%$ vs $23 \% ; 8 \%$ vs $20 \%$, respectively) ( $\mathrm{p}=0.002,0.018$, and, 0.031 , respectively) (Table 1).

In the laboratory tests, only the white blood cells (WBC) count was higher in the opium positive group $(10.1 \pm 6.60$ vs $7.38 \pm 4.14)(\mathrm{p}=0.018)$. The segmented cells and lymphocyte were higher and lower in the opium positive group, respectively (0.001 and 0.048) (Table 2).

In Table 3, the initial therapies of both groups are shown. In the opium positive group, there was a significantly lower rate of azithromycin and lopinavir/ritonavir prescription, with $\mathrm{P}$ values of 0.038 and 0.012 , respectively.

\section{Discussion}

There are overall agreements about the adverse and destructive effect of smoking in COVID-19 infected patients and about some of the controversies about opium addiction. Some studies have proposed that opium deregulates the immune system, such as increasing cytokine secretion, especially interleukin-6 $(5,7)$.

Prolonged use of opium ,by influencing the respiratory system, predisposes the addicts to structural lung disease, dysfunctional immune system, and failure of respiratory support and compensation $(5,8)$.On the other hand, addicts have more unprotected exposures in more crowded areas and even encounter more viral loads as well as more unknown or uncontrolled underlying disorders that can 
Table 1.Disease and patients' characteristics in patients with positive and negative history of opium addiction

\begin{tabular}{|c|c|c|c|}
\hline Variable & $\begin{array}{c}\text { OPIUM (positive) } \\
(\mathrm{n}=36)\end{array}$ & $\begin{array}{l}\text { OPIUM (negative) } \\
\quad(\mathrm{n}=513)\end{array}$ & $\mathrm{p}$ \\
\hline Gender (female) & $6(16.7 \%)$ & $230(44.8 \%)$ & 0.001 \\
\hline Age & $60 \pm 14.18$ & $59.31 \pm 16.55$ & 0.793 \\
\hline Hospitalization Days & $7.95 \pm 8.39$ & $8.35 \pm 5.11$ & 0.771 \\
\hline ICU Days & $2.36 \pm 3.81$ & $0.86 \pm 2.90$ & 0.026 \\
\hline ICU ADDMISSION & $13(36.1)$ & $58(11.3)$ & 0.005 \\
\hline PCR (positive) & $4(11.1 \%)$ & $53(10.3 \%)$ & 0.781 \\
\hline chills (yes) & $15(41.7 \%)$ & $330(64.3 \%)$ & 0.106 \\
\hline Fever (positive) & $14(38.9 \%)$ & $338(65.9 \%)$ & 0.002 \\
\hline Fever length & $1.50 \pm 2.50$ & $2.45 \pm 3.50$ & 0.143 \\
\hline Dyspnea (positive) & $22(66.7 \%)$ & $347(68 \%)$ & 0.870 \\
\hline Fatigue (positive) & $22(75.9 \%)$ & $368(72.6 \%)$ & 0.700 \\
\hline Anorexia (positive) & $17(63 \%)$ & $310(63 \%)$ & 0.996 \\
\hline Body pain (positive) & $16(61.5 \%)$ & $305(59.8)$ & 0.861 \\
\hline Diarrhea (positive) & $6(23.1 \%)$ & $94(18.7 \%)$ & 0.582 \\
\hline New Diarrhea (positive) & $3(11.5 \%)$ & $49(9.8 \%)$ & 0.773 \\
\hline Sore through (positive) & $3(11.5 \%)$ & $113(22.3 \%)$ & 0.115 \\
\hline $\mathrm{N} \& \mathrm{~V}$ (positive) & $10(27.8 \%)$ & $181(35.3 \%)$ & 0.345 \\
\hline Sputum (positive) & $8(32 \%)$ & $134(26.4 \%)$ & 0.540 \\
\hline Chest discomfort (positive) & $7(26.9 \%)$ & $161(31.8 \%)$ & 0.606 \\
\hline Headache (positive) & $8(29.6 \%)$ & $175(34.6 \%)$ & 0.598 \\
\hline Vertigo (positive) & $4(16 \%)$ & $122(24.2 \%)$ & 0.300 \\
\hline Delusion (positive) & $3(12 \%)$ & $52(11.1 \%)$ & 0.891 \\
\hline LOC (positive) & $9(30 \%)$ & $84(17.8 \%)$ & 0.169 \\
\hline Anosmia or hyposmia (positive) & $2(8 \%)$ & $108(22.7 \%)$ & 0.018 \\
\hline Dysgeusia (positive) & $2(8 \%)$ & $101(19.7 \%)$ & 0.031 \\
\hline Heart Disease (positive) & $9(30 \%)$ & $129(25.2 \%)$ & 0.562 \\
\hline Lung Disease (positive) & $5(13.9 \%)$ & $56(10.9 \%)$ & 0.584 \\
\hline Kidney Disease (positive) & $6(21.4 \%)$ & $48(9.5 \%)$ & 0.146 \\
\hline Dialyzed (positive) & $3(10.7 \%)$ & $15(3 \%)$ & 0.209 \\
\hline \multicolumn{4}{|l|}{ Immunodeficiency (positive) } \\
\hline DM (positive) & $12(3 \overline{7} .5 \%)$ & $156(\overline{30} .6 \%)$ & $0 . \overline{4} 17$ \\
\hline HTN (positive) & $8(26.7 \%)$ & $152(30.1 \%)$ & 0.691 \\
\hline Malignancy (positive) & $3(10.7 \%)$ & $24(4.7 \%)$ & 0.328 \\
\hline Variable & $\begin{array}{c}\text { OPIUM (positive) } \\
(\mathrm{n}=36)\end{array}$ & $\begin{array}{c}\text { OPIUM (negative) } \\
(\mathrm{n}=513)\end{array}$ & $\mathrm{p}$ \\
\hline WBC & $10.1 \pm 6.60$ & $7.38 \pm 4.14$ & 0.018 \\
\hline Diff segment & $73 \pm 20.47$ & $56.5 \pm 32.60$ & 0.001 \\
\hline Diff lymphocyte & $15.60 \pm 8.25$ & $18.70 \pm 10.12$ & 0.048 \\
\hline $\mathrm{ESR}^{-}$ & $40 \pm 24$ & $49 \pm 26.32$ & 0.118 \\
\hline CRP & $8.16 \pm 15.32$ & $8.60 \pm 15.80$ & 0.874 \\
\hline $\mathrm{Cr}$ & $1.42 \pm 1.52$ & $1.15 \pm 0.61$ & 0.301 \\
\hline AST & $34 \pm 22.8$ & $40 \pm 28$ & 0.225 \\
\hline ALT & $24 \pm 19.95$ & $27 \pm 31.3$ & 0.659 \\
\hline LDH & $618 \pm 262$ & $613 \pm 260$ & 0.923 \\
\hline CPK & $227 \pm 343.6$ & $208 \pm 318$ & 0.781 \\
\hline \multicolumn{4}{|c|}{ Table 3.Initial therapies during hospitalization in patients with positive and negative history of opium addiction } \\
\hline Variable & $\begin{array}{c}\text { OPIUM (positive) } \\
(\mathrm{n}=36)\end{array}$ & $\begin{array}{l}\text { OPIUM (negative) } \\
(\mathrm{n}=513)\end{array}$ & $\mathrm{p}$ \\
\hline Azithromycin & $7(19.4 \%)$ & $178(34.7 \%)$ & 0.038 \\
\hline Heparin & $29(80.6 \%)$ & $383(74.7 \%)$ & 0.403 \\
\hline Lopinavir and ritonavir & $17(47.2 \%)$ & $359(70 \%)$ & 0.012 \\
\hline Linezolid & $10(27.8 \%)$ & $114(22.2 \%)$ & 0.408 \\
\hline Hydroxyl chloroquin & $33(91.7 \%)$ & $453(88.3 \%)$ & 0.811 \\
\hline
\end{tabular}

justify the results of our study about more severe disorders and worse outcomes, although we could not discuss infection risk or mortality rate because of some limitations.

In line with our study, the study of Wang et al showed an increased risk of infection and mortality rate in patients with addiction compared with general COVID-19 patients, and this negative effect was seen more in AfricanAmerican patients compared to Caucasians; the differences have been proposed for probable underlying disorders in various ethnic groups (8). The results of our study support the results of the latter study regarding disease severity but since we did not evaluate mortality rate, we cannot discuss this entity.

There are some controversies about the effect of addiction on COVID-19 like its protective or triggering role, so that there are few evidences that emphasize the protective role and the other evidences against any protective role and have reported addiction as a triggering or worsening factor of COVID-19 (Khoshab, Saeedi, Wang) (4, 5, 8).

We found some interesting results, including more fehttp://mjiri.iums.ac.ir

Med J Islam Repub Iran.2021 (8 Sep); 35.115. 
brile patients, anosmia/hyposmia and dysgeusia presentations, and leukocytosis with more segmented cell ratios at the initiation of hospitalization in patients with addiction, which could be related to structural damage of their respiratory system or more susceptibility to additional bacterial superinfections.

The authors of this study have worked with great efforts on various aspects of COVID-19 (9-22), and tried to report the results of this study to evaluate the effects of opium addiction in patients with COVID-19. They also found a significant association between more severe features of COVID-19 and opium addiction.

\section{Limitations and Recommendations}

In this study, we did not have a control group to evaluate the probable protective or triggering effect of opium in the infection rate of COVID-19. Also, we did not consider mortality in our outcomes, as we retrospectively collected the data of opium addiction of our discharged patients and did not gather the data of patients who died during hospitalization. In this study, the clinical and imaging severity scores of patients were not calculated based on predefined scores because during the data collection these scores were not as popular as are now, and the main outcomes of this study for comparison between 2 groups, which were considered indirectly to be associated with the overall severity score of the disease, were the mean days of hospitalization, the necessity for ICU admission, the average days of staying in ICU, and the need to take second-line therapeutic options. Unfortunately, we did not find any national study about overall opium addiction prevalence in all age groups of the Iranian population to even theoretically discuss the effect of opium on the infection rate of COVID-19 in Iranian patients. Moreover, we did not focus on positive smoking history or type of substance that the patients with addiction used to abuse, we just took a history of any addiction or opium usage. Certainly, the type of addiction and route of substance abuse may impact the subgroup dada analysis. However, at first, we chose the most accessible, fastest, and simplest data gathering route. Thus, we recommend conducting well-designed controlled studies to obtain more explicit results.

\section{Conclusion}

Opium-addicted patients with COVID infection may be more febrile and experience more disease-specific symptoms and more severe disease course. These patients may reveal more evidence of laboratory inflammation and probable superinfections, so they should be managed with more care and with different or additional therapeutic regimen.

\section{Acknowledgement}

The authors would like to thank Rasool Akram Medical Complex Clinical Research Development Center (RCRDC) for its technical and editorial assists.

\section{Conflict of Interests}

The authors declare that they have no competing interests.

\section{References}

1. Li X, Geng M, Peng Y, Meng L, Lu S. Molecular immune pathogenesis and diagnosis of COVID-19. J Pharm Anal. April 2020;10(2):102-108.

2. Vardavas CI, Nikitara K. COVID-19 and smoking: A systematic review of the evidence. TobInduc Dis. 2020 Mar 20;18:20.

3. Reddy RK, Charles WN,Sklavounos A. The effect of smoking on COVID-19 severity: A systematic review and meta-analysis. J Med Virol. 2020:1-12.

4. Khoshab H, Rajabalipour M. The Relationship between Opium Use and Coronavirus Infection, A Brief Research Report from Iran. Medicine. 2020.

5. Saeedi M, Omrani-Nava V, Maleki I, Hedayatizadeh-Omran A, Ahmadi A, Moosazadeh M, et al. Opium Addiction and COVID-19: Truth or False Beliefs. Iran J Psychiatry Behav Sci. 2020;14(2):2.

6. Prokop M, van Everdingen W, van Rees Vellinga $T$, van Ufford HQ, Stöger L, Beenen L, et al. CO-RADS: A Categorical CT Assessment Scheme for Patients Suspected of Having COVID-19Definition and Evaluation. Radiology. 2020;296:2:E97-E104.

7. Nabati S, Asadikaram G, Arababadi MK, Shahabinejad G, Rezaeian M, Mahmoodi M, et al. The plasma levels of the cytokines in opiumaddicts and the effects of opium on the cytokines secretion by their lymphocytes. Immunol Lett. 2013;152(1):42-6.

8. Wang QQ, Kaelber DC, Xu R. COVID-19 risk and outcomes in patients with substance usedisorders: analyses from electronic health records in the United States. Molecular Psychiatryhttps://doi.org/10.1038/s41380-020-00880-7. Published online: 14 September 2020.

9. Seirafianpour F, Mozafarpoor S, Fattahi N, Sadeghzadeh-Bazargan A, Hanifiha M, Goodarzi A. Treatment of COVID-19 with pentoxifylline: Could it be a potential adjuvant therapy? [published online ahead of print, 2020 May 30]. Dermatol Ther. 2020:e13733.

10. Seirafianpour F, Sodagar S, Pour Mohammad A, Panahi P, Mozafarpoor S, Almasi S, et al. Cutaneous manifestations and considerations in COVID-19 pandemic: A systematic review [published online ahead of print, 2020 Jul 8]. Dermatol Ther. 2020; 13986

11. Nobari NN, Goodarzi A. Patients with specific skin disorders who are affected by COVID-19: What do experiences say about management strategies? A systematic review [published online ahead of print, 2020 Jun 18]. Dermatol Ther. 2020; e13867.

12. Mohamadi MM, Goodarzi A, Aryannejad A, Fattahi N, AlizadehKhoei M, Miri S, et al . Geriatric challenges in the new coronavirus disease-19 (COVID-19) pandemic: A systematic review. Med J Islam Repub Iran. 2020;34(1):841-848.

13. Faiz SHR, Riahi T, Rahimzadeh P, Nikoubakht N. Commentary: Remote electronic consultation for COVID-19 patients in teaching hospitals in Tehran, Iran. Med J Islam Repub Iran. 2020;34(1):217218.

14. Atefi NS, Behrangi E, Mozafarpoor S, Seirafianpour F, Peighambari S, Goodarzi A. N acetylcysteine and coronavirus disease 2019: May it work as a beneficial preventive and adjuvant therapy? A comprehensive review study. J Res Med Sci. 2020;25:109.

15. Sadeghzadeh-Bazargan A, Behrangi E, Goodarzi A. Systemic retinoids in the COVID-19 era - are they helpful, safe, or harmful? a comprehensive systematized review study. Iran J Dermatol. 2020;23(Supp.1):S9-12.

16. Sadeghzadeh-Bazargan A, Behrangi E, Goodarzi A. Cytokine storm and probable role of immunoregulatorydrugs in COVID-19: A comprehensive review study. Iran J Dermatol. 2020;23(Supp.1):S1318.

17. NajarNobari N, Seirafianpour F, Mashayekhi F, GoodarziA.A systematic review on treatment-related mucocutaneous reactions in COVID-19patients. Dermatol Ther. 2020:e14662.

18. Mohamadi M, Fattahi N, Goodarzi A, Alizadeh-Khoei M, MiriSh, Hekmat H, et al. A Comprehensive Review on COVID-19 Infection and Comorbidities of Various Organs. Acta Med Iranic. 2021;59(1):414.

19. Najar Nobari N, Montazer F, Seirafianpour F, Nikkhah F, Aryanian Z, Goodarzi A. Histopathologic Changes and Cellular Events of Organs Systems in COVID-19. J Cell MolAnesth. 2021;6(1):81-88.

20. Kooranifar S, Sadeghipour A, Riahi T, Goodarzi A, Tabrizi S, Davoody N. Histopathologic survey on lung necropsy specimens of 15 patients who died from COVID-19: A large study from Iran with a high rate of anthracosis. Med J Islam Repub Iran. 2021;35(1):481-490. 
21. Najar Nobari N, Seirafianpour F, Dodangeh M, SadeghzadehBazargan A, Behrangi E, Mozafarpoor S, et al. A systematic review of the histopathologic survey on skin biopsies in patients with Corona Virus Disease 2019 (COVID-19) who developed virus or drug-related mucocutaneousmanifestations. Exp Dermatol. 2021 May 11. doi: 10.1111/exd.14384. Online ahead of print.

22. Sadeghzadeh-Bazargan A, Rezai M, NajarNobari N, Mozafarpoor S, Goodarzi A. Skin manifestations as potential symptoms of diffuse vascular injury in critical COVID-19 patients. J Cutan Pathol. doi: 10.1111/cup.14059. Online ahead of print. 